\title{
Archéopages
}

Archéopages Archéologie et société

\section{Des vivants et des morts. Débat}

Propos recueillis par Catherine Chauveau

Michel Tarpin, Michel Signoli et Catherine Chauveau

\section{(2) OpenEdition}

Journals

Édition électronique

URL : https://journals.openedition.org/archeopages/556

DOI : $10.4000 /$ archeopages. 556

ISSN : 2269-9872

Éditeur

INRAP - Institut national de recherches archéologiques préventives

\section{Édition imprimée}

Date de publication : 1 septembre 2014

Pagination : 82-89

ISSN : 1622-8545

Référence électronique

Michel Tarpin, Michel Signoli et Catherine Chauveau, «Des vivants et des morts. Débat », Archéopages [En ligne], 39 | 10/2013-01/2014, mis en ligne le 01 janvier 2016, consulté le 21 janvier 2022. URL: http://journals.openedition.org/archeopages/556 ; DOI : https://doi.org/10.4000/archeopages.556 


\section{Des vivants et des morts}

De la nécessité de déshumaniser l'adversaire pour l'affronter à celle de l'humaniser pour recouvrer sa dignité, il y a des variations d'attitudes multiples. Ce qui reste, c'est la quantité anormale de morts, blessés, prisonniers que génère la guerre. Comment une population, un groupe humain, s'y adaptet-elle? Quels comportements, quels rituels, quels cadres vont permettre de gérer cette situation de crise?

\section{Michel Tarpin}

est professeur d'histoire de l'art et d'archéologie des mondes antiques à l'université de Grenoble, et dirige le Centre de Recherche en Histoire et histoire de l'art - Italie, Pays Alpins. Un de ses thèmes de recherche est la définition des catégories de butin et la procédure des rituels de victoire. Il a publié notamment : «La guerre dans le monde romain », in Thesaurus Cultus et Rituum Antiquorum (ThesCRA), VIII, Private space and public space polarities in religious life. Religious interrelations between the Classical World and neighbouring civilizations, Bâle,

Fondation pour le Lexicon Iconographicum Mythologiae Classicae /The J. Paul Getty Museum, 2012, p. 223-245; «Les Manubiae dans la procédure d'appropriation du butin », in M. Coudry, M. Humm (éd.), Praeda. Butin de guerre et société dans la Rome républicaine / Kriegsbeute und Gesellschaft im republikanischen Rom (Collegium Beatus Rhenanus, 1), Stuttgart, Franz Steiner, 2009, p. 81-102; «Devenir riche par le butin : données quantitatives dans l'empire romain », in C. Baroin, C. Michel (éd.), Richesse et société, Colloques de la MAE 9, Paris, 2013, p. 66-8o.

\section{Michel Signoli}

dirige le laboratoire « Anthropologie bioculturelle, Droit, Ethique \&Santé ». Ses thèmes de recherche sont les sépultures multiples en relation avec les épidémies du passé et les conflits récents. Parmi ses dernières publications : avec T. Vette, O. Dutour, Y. Ardagna, Les oubliés de la Retraite de Russie; Vilna 1812 - Vilnius 2002, Librairie historique F. Teissedre, Paris, 2008 ; avec G. De Vedrines, « Burials Related to Recent Military Conflicts: Case Studies from France », in N. Marquez Grant and L. Fibiger (ed.) Archaeological Human Remains and Legislation:An International Guide to Laws and Practice in the Excavation and Treatment of Archaeological Human Remains, Routledge, 2010 ; avec F. Lagrange, E. Robbe, T. Vette, Les Ombres de l'Empire : approches anthropologiques, archéologiques et historiques de la Grande Armée, Cahiers d'études et de recherches du Musée de l'Armée, hors-série n 5, 2012; avec T. Vette, A. Homann, K. Sczech et S. Bock, « De la Campagne de Russie à la Campagne d'Allemagne: quatre exemples d'apports de l'archéologie et de l'anthropologie funéraire à nos connaissances sur le Ier Empire », idem, p.61-82. 


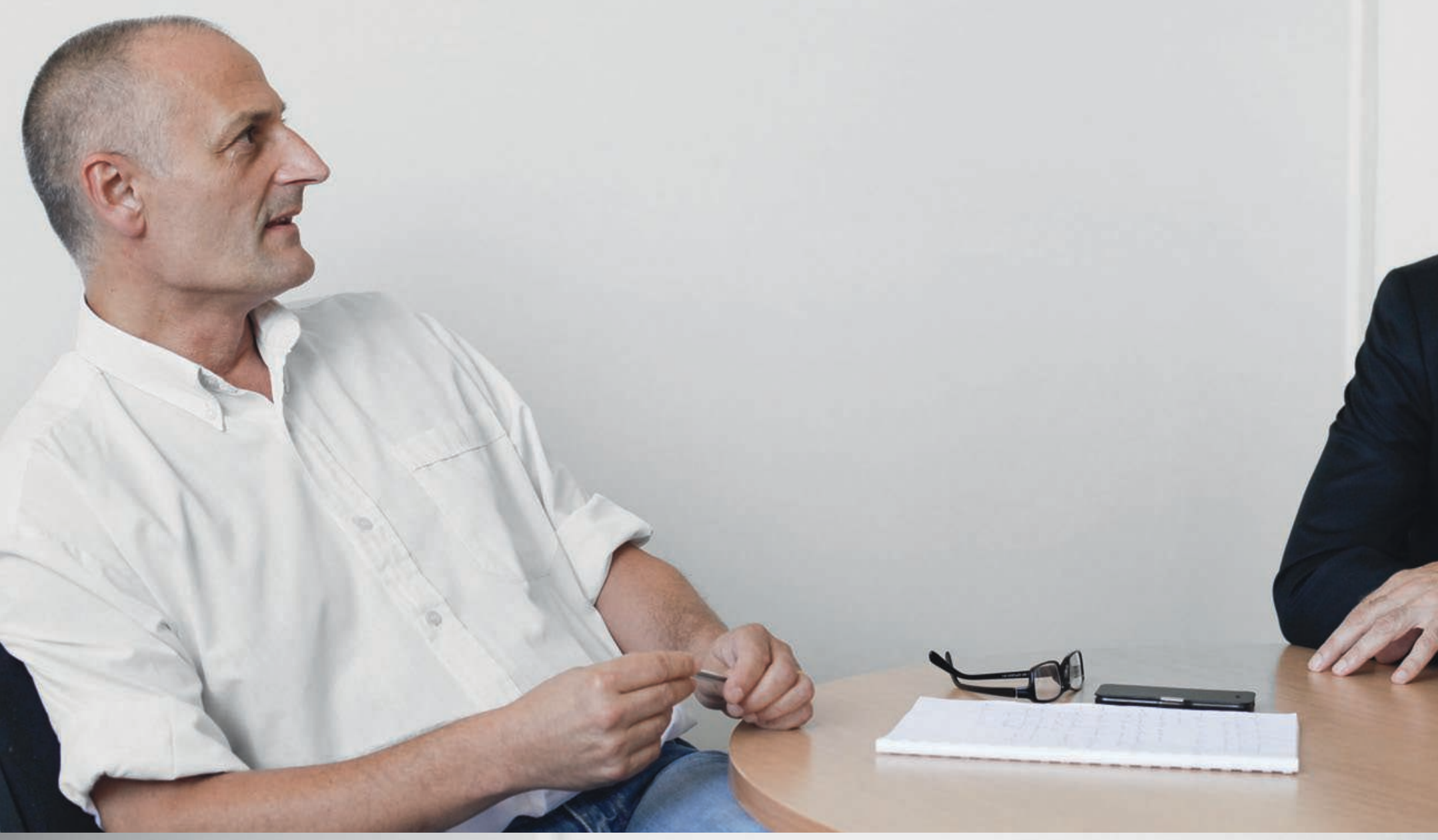


Michel Tarpin Le statut des vaincus n'est pas réellement étudié pour le monde romain. Dans une répétition des schémas des historiens précédents, on a pris l'habitude d'examiner ce genre de question en termes de droit, avec une approche juridique, et non en termes de comportement, avec une approche sociologique. Les romanistes perçoivent le plus souvent la guerre comme une technique et se focalisent donc sur les cartes d'état-major, les déplacements des troupes, les questions d'armement, etc. Le poids monumental et fascinant des études historiques allemandes consacrées à la carrière et à la stratégie y est pour quelque chose. Mais dans les textes, on ne trouve pas de droit de la victoire, de la guerre, formalisé tel qu'il peut l'être de nos jours ! Et ce que l'on peut déduire de ce qui est mentionné révèle un système assez souple. La question des captifs est, elle, parfois évoquée dans les textes latins, car elle est un élément du sujet plus vaste et majeur qu'est l'acquisition du butin présenté au triomphe à Rome, rituel de clôture de guerre. C'est durant les actions et les gestes rituels de victoire que se joue le sort des vaincus. Le premier moment qui marque le basculement du combat vers la victoire est celui où le premier soldat entrant dans la ville ennemie crie: urbs capta - ville prise, en latin. Et c'est toujours cette même expression qui se crie au Moyen Âge lors des prises de ville. Les défenses de la ville sont alors rompues en force. Sur un champ de bataille ouvert, léquivalent du rempart est la première ligne ennemie. Les corps s'accumulent dans un bain de sang jusquà ce qu'une des lignes se défasse. Rompre une ligne équivaut à rompre un rempart ou des portes et c'est cela la victoire. Si l'ennemi vous ouvre ses portes, accepte sa défaite, opte pour la reddition, il doit le faire avant l'assaut. Cela ne relève pas du droit romain, mais du droit des gens, on pourrait dire que c'est dans l'ordre des choses. Une fois la ville prise, une partie de la troupe est envoyée dans la ville tuer, violer, piller. Mais l'affectation des différentes catégories de butin aux différentes catégories de combattants est faite au préalable. Les soldats ont droit à ce qu'il reste. L'autorité militaire, voire l'autorité politique, ont un regard sur ce que l'on peut prendre ou pas, comme aux époques modernes, d'ailleurs ; le droit de pillage donné par Napoléon est précisément délimité. Les vaincus épargnés sont autorisés à sortir de leur ville avec au moins un vêtement ou un pagne parce que la nudité pose problème aux Romains. En général, on dépouille les cadavres des ennemis, on trie les armes pour mettre de côté celles qui seront dans le cortège triomphal, on fait un tas du reste et on y met le feu. Le bronze, qui compose une grande part de l'armement, fond et se transforme en masses récupérables, transportables... On n'en a pas trace mais on peut supposer que certaines offrandes de victoire sont faites à partir du métal récupéré des armes fondues. Il est probable, et c'est une hypothèse personnelle, que c'est lors de cette étape, que se règlent certaines questions comme l'élimination de certains vaincus, les invendables que sont les malades et vieillards, ou bien la limitation du nombre de captifs vivants de façon à ce qu'il soit à la fois rentable et gérable. Alors que ce type de question est abordée communément par les contemporanéistes quand ils parlent de la traite d'esclaves, ces sujets ont été « étouffés » par les antiquisants qui ont tendance à survaloriser les cultures gréco-romaines pour échapper au primitivisme. Ce massacre, caiedes en latin, est décrit comme un défoulement, une extermination, et s'arrête quand les soldats sont « rassasiés ». Cela vise tout autant à anéantir une communauté, même s'il n'est pas question de tuer tout le monde, quà en tirer le meilleur profit. Les vaincus ne sont pas censés se défendre à cette étape, ils doivent se plier à leur situation de victimes. Mais bien sûr, cela ne se passe pas toujours comme ça, au grand étonnement des Romains : certains continuent à combattre et certains se suicident en masse, habitude espagnole surtout, un peu gauloise aussi. A-t-on des études sur la psychologie de ces massacreurs et de leurs victimes faites sur les survivants des épisodes récents de génocide?

Michel Signoli Pas à ma connaissance. Mais cela mévoque quelques faits de guerre emblématiques, comme le suicide de la population de lîle japonaise de Saipan avant l'arrivée des Américains : des civils se sont massivement jetés du haut des falaises. Quant aux exterminations récentes, que ce soit au Rwanda, en Bosnie ou pendant la Seconde Guerre mondiale, on est dans des logiques d'éliminer jusquà la génétique de groupes humains donc on tue tout le monde, on terrorise pour tuer plus facilement, pour limiter les actes de résistance; et si on épargne des femmes, c'est pour les violer afin que leurs enfants soient ceux des vainqueurs. Cette négation va parfois jusquà traiter les corps de ces indésirables comme des matières premières. Cet extrémisme n'est pas généralisé dans un conflit « normal » et les codes et règlements militaires récents sont censés limiter les actes de déshumanisation des prisonniers et des cadavres.

MT Il me semble que traiter son adversaire comme un égal ou comme du bétail dépend de la situation et des étapes de la bataille. Assez régulièrement des duels précèdent le combat antique. Dans le duel, il y a comme prémisse de reconnaître l'adversaire comme un égal, sinon le duel n'a plus de sens. Tandis que lorsque vous prenez une ville et que vous massacrez ses habitants, c'est du bétail. L'hypothèse de certains chercheurs américains et que demander à des milliers de types de charger à froid est vraiment difficile. Il faut un conditionnement. Je pense que le duel fait partie de stratégies d'excitation et ne relève pas, comme on pourrait le croire, du domaine de présage. Dans la bataille rangée, il est hors de question qu'un soldat sorte du rang pour aller attaquer seul un ennemi; il n'a pas le droit de quitter sa ligne pour ne pas l'affaiblir et le combat en ligne est un acte collectif. Lorsque Marius doit affronter les Teutons, alors qu'il a moins d'hommes, il commence par maintenir ses soldats au camp, en les privant d'eau et en leur interdisant de répliquer aux provocations des Teutons. Et quand ils sont bien énervés, il les lâche... et remporte la victoire ${ }^{1}$ ! Une fois cette adversité et cette altérité installées, l'ennemi vivant ou mort, peut passer au statut d'objet dans le discours et dans les faits. Dans le monde romain, les captifs, ceux
1. La bataille d'Aix, en 102 avant notre ère, relatée par Plutarque dans Vie de Marius 


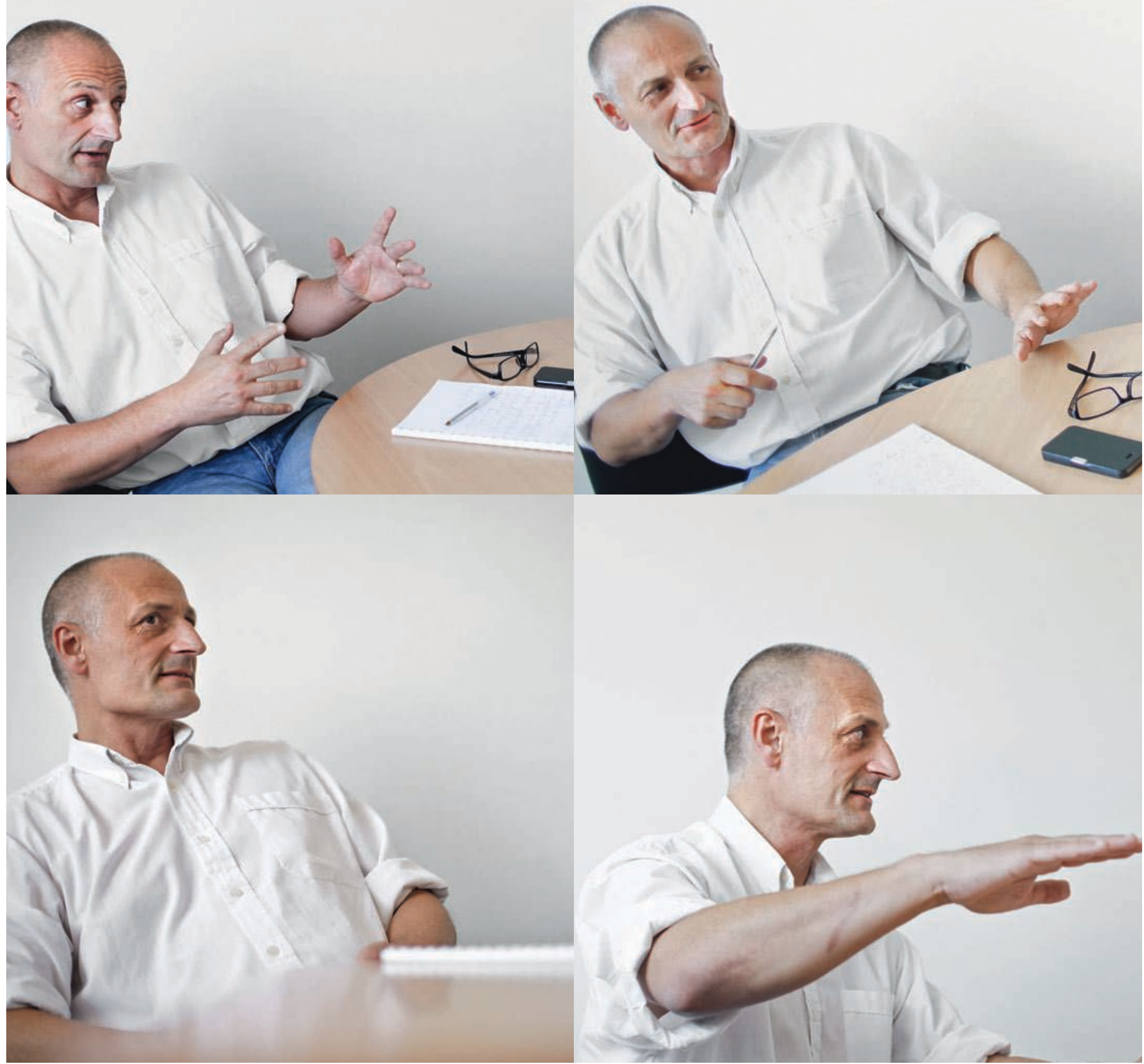

La mémoire des lieux d'inhumation en lien avec un épisode traumatique se perd dans la très grande majorité des cas. 
qui n'ont pas été tués sont réduits à létat de marchandises, avec une nuance cependant: Tite-Live différencie les captifs libres (étonnant concept!), ceux qui avaient un statut de citoyen auparavant, et les captifs esclaves, anciens esclaves que les soldats ont le droit de s'approprier lors du pillage, comme d'autres objets, alors que les premiers, propriété du peuple romain, font partie du butin et doivent être protégés. Quant aux cadavres, il y a peu de cas mentionnés où ils sont traités comme des objets : celui de la bataille de Munda, en Espagne, où César luttant contre les derniers Pompéiens, utilise les corps pour rehausser le terrain au pied des remparts tout en faisant brandir les têtes sur des piques ; celui du massacre des Teutons à Aix-en-Provence, en 102, où l'on raconte qu'il y eut tant de tués que les gens du coin ont utilisé les os pour faire la clôture de leurs vignobles. Sur ce besoin d'enlever à l'autre le statut d'être humain, la situation la plus délicate n'est-elle pas le contexte de guerre civile? Il faut trouver à ceux qui ont le même statut que soi, qui appartiennent à la même communauté, quelque chose qui les place dans un ailleurs où il sera permis d'adopter des comportements réservés aux barbares. C'est le cas de la prise de Crémone par Vitellius décrite par Tacite ${ }^{2}$. Les armées romaines revenant de Germanie, prennent une ville italienne qui avait accueilli-mais elle n'avait pas le choix les troupes ennemies. Massacrer ces traîtres est loccasion d'un énorme défouloir des soldats.

MS Il est sûr que les contextes de guerre civile déclenchent des extrémismes comportementaux. Lextermination de groupes perçus comme indésirables au sein d'un pays à cause de leur religion, culture, position politique etc. est marquée notamment par une forte volonté d'oubli. Les exemples que nous avons en France sur les périodes modernes ne sont pas extrêmement nombreux. Il y a les fosses des tués de la Virée de Galerne, au Mans, cas très intéressant étudié par Elodie Cabot ${ }^{3}$. Mais on n'a rien concernant les guerres de religion, par exemple. Par contre, lactualité mondiale plus récente nous en a donné de nombreux exemples. Notre laboratoire est intervenu en Espagne, sur des contextes d'inhumations d'exception qui font suite à des exécutions sur des territoires occupés par l'armée franquiste, dont le but est de terroriser des populations pourtant déjà sous contrôle militaire. Les groupes d'exécuteurs prennent position dans les villages avec leur liste de victimes (des hommes, en général dans la force de lâge et considérés comme de potentiels adversaires politiques) et leur matériel. Les victimes sont exécutées, inhumées sommairement dans des tranchées creusées à l'avance. Il n'y a aucun traitement des corps : ils sont comme ils étaient au moment de l'exécution, avec leurs vêtements et ce qu'ils avaient en poches. Cela ne relève pas d'un respect, mais bien d'une mise à distance de type : « Ils n'ont rien à voir avec nous et il n'y a rien de bon à prendre chez eux ». Mais ils sont couverts de chaux, un moyen bien connu d'accélérer la décomposition des chairs. Et bien sûr, aucun marquage du lieu. Plusieurs de mes collègues qui travaillent sur des situations de violence de masse du $\mathrm{XX}^{\mathrm{e}}$ siècle, en Europe centrale, en Amérique du Sud etc. constatent cette obsession de la disparition des corps qui peut s'interpréter à la fois comme une négation de la personne tuée et comme une négation de l'acte de tuer. MT Il y a un certain nombre d'interdits liés à la guerre civile antique : on ne triomphe pas, on ne célèbre pas. Il y a un fait anthropologiquement intéressant, en 63 avant notre ère ${ }^{4}$, à la suite du massacre des troupes de Catilina: on demande aux soldats de rentrer au camp en silence, les armes nettoyées comme s'il ne s'était rien passé. La situation est ramenée à une sorte de zéro. Normalement on rentre aux camps avec les épées maculées de sang comme preuve, armes qui du coup ne pourront plus resservir car le sang corrode très vite le fer. Après la bataille de Modène, où Octavien bat Antoine, Cicéron demande un monument spécifique pour les vainqueurs, mais en mettant en avant le caractère exceptionnel de cette demande. Ni vainqueurs ni vaincus n'ont droit à des sépultures bien spéciales, car ce sont épisodes que l'on essaie de faire oublier le plus rapidement possible.

MS Le but est bien l'oubli le plus total possible. Avec la deuxième moitié $\mathrm{du} \mathrm{xx}^{\mathrm{e}}$ siècle, on s'aperçoit qu'il ne suffit plus aux exécuteurs de compter sur les caractéristiques de la chaux et sur l'oubli des lieux d'inhumation. Dans certains pays d'Amérique du Sud, par exemple, les exécutions se font en jetant en mer les gens lestés, du haut d'hélicoptère ou d'avion 5 .En Bosnie, on s'est rendu compte, grâce aux analyses génétiques, que les restes des individus exécutés et inhumés pouvaient se retrouver répartis en divers lieux, avec des interventions successives d'inhumation et d'exhumation. Connaissez-vous des exemples de ce genre de pratiques, à part les cas de décollation avec inhumation séparée des corps et des têtes?

MT La disparition par immersion est fréquente dans l'Antiquité: on jette à l'eau tout ce qui est ingérable socialement car l'eau est un lieu d'exterritorialité. Mais dans l'Antiquité, on préfère faire disparaitre la mémoire : l'identité de l'individu étant concentrée dans la tête réelle ou représentée, donc dans le portrait, et dans le nom, on fait disparaître et interdire l'un et l'autre même, stratégie que les Soviétiques ont manipulée avec beaucoup de talent. C'est dans la même idée que l'on peut décapiter un mort, pour faire en sorte que le corps et la tête ne puissent jamais être reliés. La destruction violente et rapide de la personne peut aller plus loin : lorsque la populace de Rome s'en prend à certains empereurs, on insulte le corps, on le frappe, on le décapite voire le démembre, on le traîne avec des crocs de boucher et on le jette... dans le Tibre!

MS Cela me fait penser aux restes d'Hitler, récupérés par Staline, puis transportés, cachés et finalement jetés dans une rivière... Pour Staline, détenir cette caisse, c'était s'affirmer comme le vainqueur. La disparition des restes empêche que quelqu'un d'autre ne les récupère à son profit, d'une manière ou d'une autre. Au Rwanda comme en Bosnie, l'énormité du massacre a suscité deux types de réactions : l'ensevelissement rapide en fosses communes par les vainqueurs ou par les vaincus; ou bien, phénomène beaucoup plus rare, lintervention zéro. Dans certains lieux, les corps sont laissés sur place dans cadavres dans les violences

de masse et les génocides ",

Paris, 2013. 

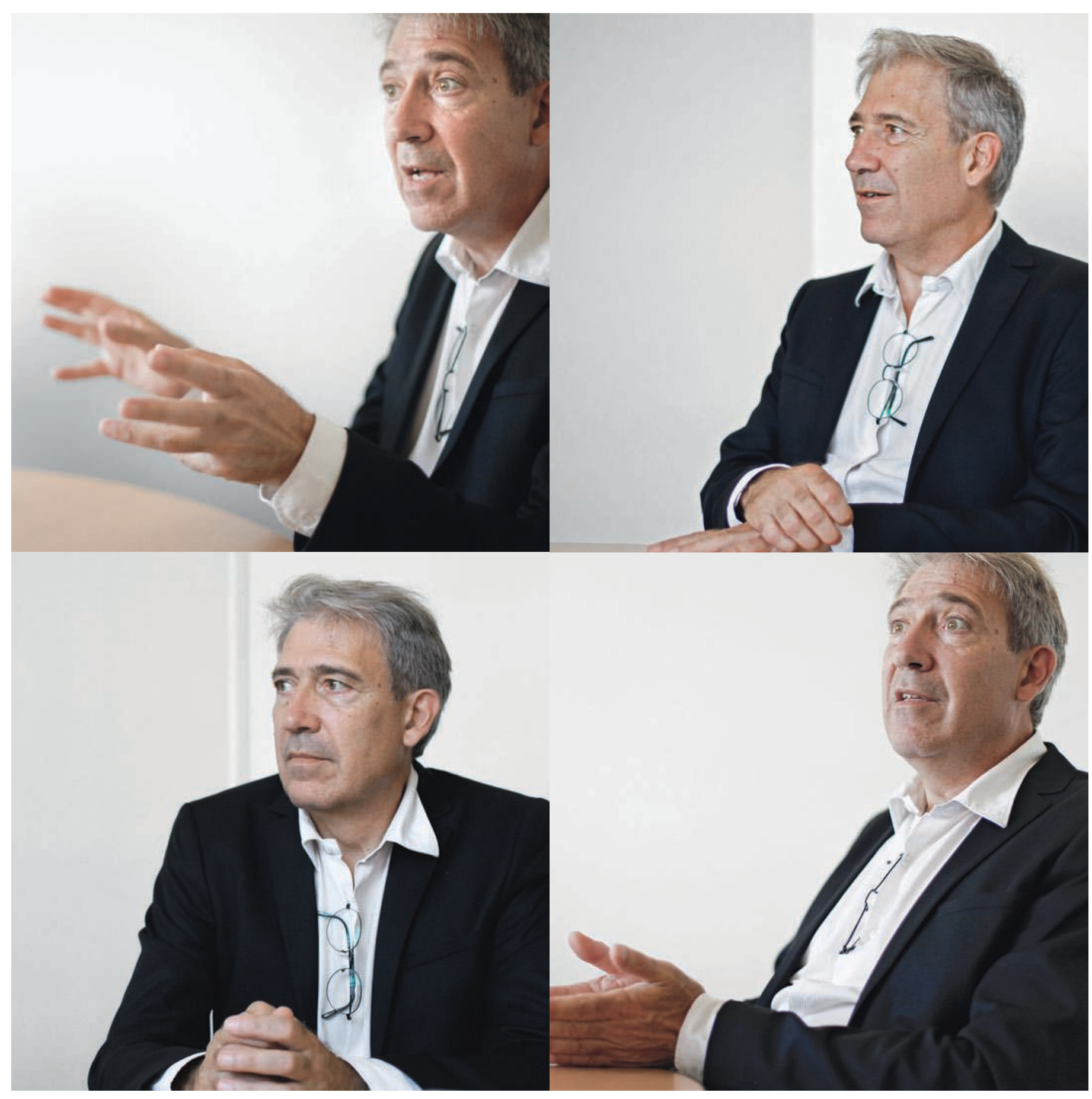

$\infty$

Les Romains sont assez hostiles à l'idée de laisser le vaincu venir enterrer ses morts, ce qui est une position très particulière dans le monde antique. 
la rue, dans des bâtiments pendant des mois et des mois. On s'explique mal pourquoi. Le comportement habituel, majoritairement adopté, est de récupérer ses morts et de les replacer en situation acceptable, si ce n'est digne. L'attitude minimale est d'ensevelir les morts en fosse commune. Depuis les conflits récents, chaque armée est censée enterrer ses morts, mais divers éléments peuvent perturber cette règle. En 1914, les premières semaines, les premiers mois, les lignes de front vont beaucoup bouger. Les cadavres sont enterrés par les soldats qui récupèrent le terrain, quelles que soient les nationalités, ou bien par les civils qui vivent là. Entre la guerre francoprussienne de 1870 et le premier conflit mondial, les mentalités vis-à-vis de la mort évoluent pour les militaires comme pour les civils : on supporte moins la mort du proche et on vit plus difficilement la disparition de son corps et l'enfouissement collectif. D'où cette idée pour certains belligérants, au début de la Première Guerre mondiale, de faire un marquage individuel du soldat, de façon à pouvoir l'identifier. Mais toutes les armées sont dépassées par l'importance de la mortalité dans les premières semaines du conflit et reviennent à la gestion des morts telle qu'elle se pratiquait au XIX ${ }^{\mathrm{e}}$ siècle, c'est-à-dire en fosse commune mais, et surtout côté français, en essayant ne pas mélanger Français et Allemands. Et dans certains cas, on constate même que les dépouilles ne sont pas traitées de façon identique. Je pense à cette fouille qui a eu lieu en 2009 à Fromelles, lieu d'un affrontement des premiers jours de la bataille de la Somme, où l'Australie s'est engagée de façon importante sur le front occidental. Les Australiens prennent une première ligne allemande, mais elle est récupérée par les Allemands au bout de quelques heures. Ce sont donc ceux-ci qui vont gérer les corps des soldats australiens tués dans la tranchée. Les corps ont été enterrés superposés, en rangées, dans des fosses. Ils sont déséquipés (on leur a retiré armes, casques...) et plus de la moitié sont en décubitus dorsal (face contre terre). Selon moi, cela dénote une volonté de punir, d'humilier, ces ennemis.

MT Il est sûr que le traitement du corps défunt, et du corps ennemi, est un problème, pour les Romains... et pour les historiens du monde romain qui ont oublié le corps. À la différence de nos collègues du monde grec, passés dans les années 1960 à une démarche plus sociologique de l'histoire, nous sommes centrés sur des questions plus théoriques. Pour la période romaine, on n'a ni grands cimetières militaires, ni sources précises sur ces questions. Normalement, chacun doit récupérer ses morts, même si cela se fait de manières différenciées. Mais les Romains ont une position très particulière dans le monde antique : la volonté d'empêcher l'adversaire vaincu de récupérer les siens. Et ils sont très embêtés quand ils se trouvent défaits et en situation de devoir demander à reprendre leurs morts. Cela relève plutôt de leurs nombreuses craintes et superstitions. Il y a l'idée que tant qu'il reste quelque chose de concret d'un mort, on ne sait pas à quoi s'attendre : les défunts peuvent avoir des velléités de réapparaître ; les adversaires, vainqueurs ou vaincus, peuvent avoir des intentions de s'attaquer aux dépouilles des soldats romains... L'inhumation sur le champ de bataille n'apparaît alors en aucun cas comme la bonne solution et on lui préfère l'incinération. Quant à la position sur le ventre, attestée occasionnellement dans des nécropoles du monde antique, d'une façon générale, elle est censée empêcher le mort de revenir. En ce qui concerne l'équipement des ennemis, il est prélevé, détruit ou consacré aux dieux, parfois exposé chez des particuliers, mais on évite de le réutiliser, sans doute par superstition. Lorsqu'on doit y recourir, on le fait porter par des esclaves et non par des citoyens. J'ai une interrogation sur les chiffres des tués lors de ces grandes batailles antiques. Il est possible que ces chiffres soient gonflés et qu'ils n'aient rien de réalistes. Mais on parle de milliers voire de dizaines de milliers de morts par bataille. Le chiffre déclaré, par exemple, pour la bataille d'Orange en 107 avant notre ère est de 80 ooo hommes tués par les Cimbres! C'est extrêmement déroutant quand on lit les Histoires de Polybe. Il livre des chiffres d'ordres de grandeur sans aucune commune mesure concernant les batailles d'un côté entre Romains et Carthaginois ou Romains et Gaulois, et de l'autre entre Grecs. Dans le monde grec une bataille fait 150 morts, 200 morts. Mais quand Hannibal arrive en Italie, année après année, les combats font tous des milliers de morts parmi les Romains. Si on prend ces chiffres comme tels, il est clair que dès les premières grosses défaites, la génération mobilisable n'existe plus. A Rome, il y a eu des générations entières exterminées, des familles qui disparaissent purement et simplement. Quand Hannibal rentre en Afrique, il n'a plus d'armée. Et pourtant il l'a regonflée régulièrement. Larmée de son frère Hasdrubal est massacrée sur place, à part les Gaulois qui courent assez vite pour s'échapper. Hannibal est censé avoir récupéré ses morts, mais les Romains ne se sont pas occupés de leurs corps.

MS C'est très impressionnant parce que je ne vois pas comment ils ont pu incinérer ces milliers de corps ni comment il est possible que l'archéologie soit passée à côté ? Pourtant, c'est souvent dans ces zones un peu en marge de la ville antique, de la ville médiévale, de la ville moderne, zones aujourd'hui concernées par divers aménagements, qu'on va tomber sur les lieux d'inhumation massive, en contexte de crise, de corps dont il faut se débarrasser au plus vite : les pestiférés, les massacrés, les victimes de catastrophes naturelles... À Marseille, par exemple, on a fouillé deux fosses de la grande épidémie de 1720 et on en connaît trois autres. Mais où sont les morts des autres épidémies plus anviennes et parfois plus meurtrières ? Il n'est pas question de remettre en cause les chiffres des batailles ou des épidémies mais il y a vraiment une logique qui nous échappe statistiquement.

MT De toute façon, il nous manque $90 \%$ des morts du passé. Combien de temps, lorsqu'on abandonne des cadavres sur un champ de bataille, en reste t-il quelque chose de vraiment repérable?

MS C'est extrêmement variable en fonction de la saison et de la nature du sol; plus il y a d'humidité et de chaleur, plus la décomposition est rapide. En sus des vers et des micro-organismes, les cadavres sont attaqués par des rongeurs ou de plus gros prédateurs qui ne vont 


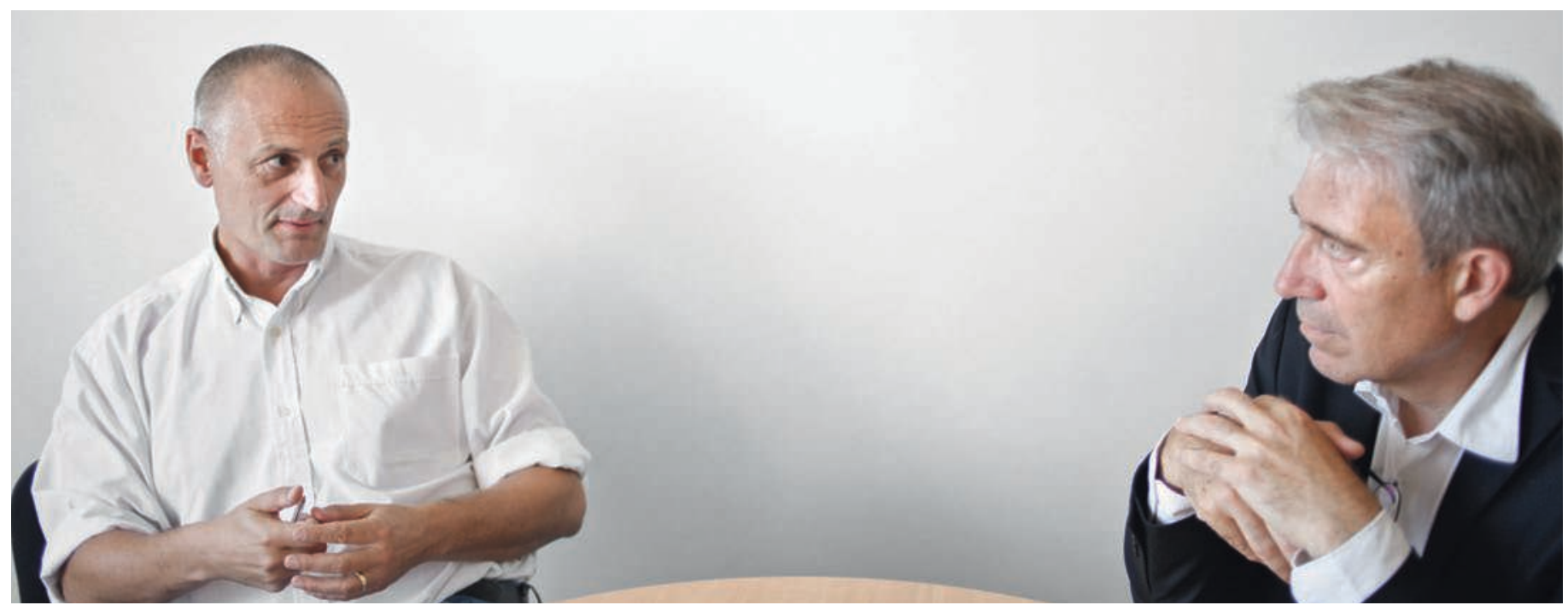

pas tout consommer ni tout emporter mais considérablement fragmenter les os. Tout cela combiné peut entraîner une disparition totale en quelques mois. Et la mémoire disparaît en quelques décennies. On ne sait plus aujourd'hui avec précision où sont les fosses de la bataille d'Austerlitz, par exemple. Il n'y a pas eu de monuments sur place, sur le moment; il n'y a qu'une exception sur le lieu de la bataille d'Eylau, où des soldats français repassant mois plus tard sur le champ de bataille nettoyé sans doute par des civils, ont planté une petite stèle à la mémoire d'un gradé. C'est après la guerre de 1870, que sont construits des cimetières militaires parfaitement identifiés avec des monuments commémoratifs, sur les lieux où ont été inhumés à la suite d'un combat les Allemands ou les Français ou les deux. En 1914, dans un premier temps, on creuse à la hâte des fosses multiples dont on perd plus ou moins la trace, mais ensuite les corps sont ramassés et inhumés en arrière des lignes de front... Ensuite on constituera de grands cimetières militaires regroupant les défunts de tout un secteur, en séparant les belligérants. Dès le lendemain de la guerre, en France, la famille et les descendants ont le choix de laisser leur parent avec ses frères d'armes dans la nécropole la plus proche du lieu du décès, ou de le transférer dans leur caveau familial. MT Cette question de ramener le corps ou pas se pose clairement dans l'Antiquité. Quelques textes indiquent que c'est probablement au début du $\mathrm{I}^{\mathrm{er}}$ siècle avant notre ère qu'on a commencé à imposer l'ensevelissement sur place car, à la suite d'une défaite, le rapatriement des cadavres à Rome avait déclenché des troubles tels que le Sénat a décidé que c'était nuisible à l'ordre public. Les soldats romains seront donc laissés sur place, de préférence incinérés et leurs urnes funéraires éventuellement rapportées à Rome. Quant aux monuments commémoratifs, avec des corps associés, c'est une coutume précoce, même sur les champs de bataille; il y a ceux de Marathon, au début du $\mathrm{V}^{\mathrm{e}}$ siècle avant notre ère; celui du Lion de Chéronée, en 338. Il ne faut pas confondre ces monuments avec les trophées proprement dits car le mot trophée est utilisé pour décrire diverses choses. Le trophée fondamentalement, c'est un pilier de bois ou un arbre débranché sur lequel on accroche une partie des armes des vaincus. Cicéron dit très clairement que c'est une coutume grecque pas une coutume romaine. La coutume proprement romaine est de construire à Rome où sur les lieux de victoire des monuments en dur visibles de très loin, comme le monument de la Turbie pour Auguste, celui du col du Perthus pour Pompée auparavant. On les appelle trophées parce que l'on n'a pas de nom particulier. Le premier de ces monuments est lié à la victoire sur les Allobroges. Quelque partà la jonction de l'Isère et du Rhône, il y a quelque chose de construit en 121 avant notre ère. D'après l'unique source qui en parle, ce serait un monument-tour orné de trophées. Il faut sans doute imaginer quelque chose d'assez proche des "mausolées" circulaires bien connus, comme celui d'Auguste. Le mieux connu est le grand mausolée d'Adamclisi, en Roumanie, pendant de la colonne Trajanne de Rome, ceint d'une frise de métopes qui illustrent les différentes batailles de Trajan autour duquel on a retrouvé des fragments de monuments gravés de listes de soldats morts. Cela ressemble un peu à nos monuments de 14-18. Mais je reste surpris par l'absence de monument, car on devrait avoir en plus. Le monument funéraire de Pharsale, connu seulement par les textes, est décrit dans des termes qui indiquent que ce devait être quelque chose de normal. L'histoire dit que César fait voter ses soldats pour l'attribution des palmes de victoire, il reçoit les deux premières, la troisième est attribuée à un centurion mort au combat, qui a mené les troupes à la victoire. César dit bien que tant de gloire vaut un monument individuel à côté du monument collectif. 